\title{
Regulatory mechanisms of immune responses to intestinal bacteria
}

\author{
$\mathrm{K}_{\text {Honda }}{ }^{1,2,3}$ and K Takeda ${ }^{1,2}$
}

The intestinal mucosa allows the residence of trillions of bacteria with which it interacts dynamically. To establish and maintain a mutually beneficial relationship, the mucosal immune system must enforce tolerance toward the vast nonpathogenic microbiota while simultaneously remaining reactive to potentially pathogenic microbes; the disruption of this delicate balance results in inflammatory bowel diseases. In this review, we describe advances in our understanding of regulatory mechanisms of the innate immune system and how these shape adaptive immune systems during steadystate and inflammatory processes in the intestinal mucosa.

\section{INTRODUCTION}

The gastrointestinal tract is composed of very unique tissue with the presence of commensal bacteria. ${ }^{1}$ Commensal bacteria colonize the intestinal lumen shortly after birth and comprise approximately 1,000 species with a density of $10^{12}$ organisms per milliliter of luminal contents in the large intestine. ${ }^{2-4}$ The host intestine provides bacteria with a microenvironment rich in nutrients derived from ingested food. Commensal bacteria, in turn, contribute to the digestion of food, provision of essential nutrients, and prevention of propagation of pathogenic microorganisms. ${ }^{5,6}$ To maintain these beneficial relationship, the host immune system should remain hypo-responsive to commensal bacteria. However, at the same time, the intestinal immune system has to combat invasive pathogenic bacteria. Thus, the immune system in the intestinal mucosa has very complicated features that are distinct from that in other tissues. The intestinal immune system mounts a strong inflammation against invasive pathogenic bacteria while providing many layers of inhibitory mechanisms not to mount excessive immune response against commensal bacteria. The breakdown of such a delicate balance of the intestinal immune system causes the development of inflammatory bowel diseases (IBDs). ${ }^{7,8}$ Accordingly, the regulatory mechanisms of immune responses to intestinal bacteria have recently been a subject of intensive research.

\section{REGULATION OFTLR SIGNALING INTHE INTESTINE}

Among some of the most impressive progress in the field of immunology in the last decade has been the elucidation of the mechanisms for innate immune recognition of microorganisms. ${ }^{9}$ Innate immunity specifically recognizes molecular patterns of microorganisms through Toll-like receptors (TLRs) and other pattern-recognition receptors, and use of pattern-recognition receptors helps regulate the development of antigen-specific adaptive immune responses. ${ }^{10-12}$ Functional characterization of TLRs has made us aware that TLR-dependent responses at the intestinal mucosa should be finely regulated. Macrophages isolated from the intestinal lamina propria of healthy humans and mice have been shown to be hypo-responsive to TLR stimulation. ${ }^{13-16}$ In contrast, substantial amounts of TLR-dependent cytokine production can be observed in intestinal lamina propria macrophages from hosts with intestinal inflammation. ${ }^{13,14}$ For example, macrophages from interleukin (IL)-10-deficient mice and myeloid cell-specific Stat3-deficient mice, both of which spontaneously develop intestinal inflammation, are in a state of constitutive and aberrant activation. ${ }^{17}$ In these mutant mice, blockade of the TLR signaling by the introduction of deficiency in TLR4 or MyD88 abrogates the development of intestinal inflammation, ${ }^{18,19}$ indicating that TLR-dependent excessive inflammatory responses at the intestinal mucosa causes development of colitis. In addition, as with the murine model of intestinal inflammation, a unique subset of macrophages with excess TLR-dependent inflammatory responses were observed in the lamina propria of IBD patients. ${ }^{20-22}$ Thus, negative regulation of TLR-dependent responses in intestinal lamina propria macrophages is a prerequisite to prevent intestinal inflammation both in humans and in mice.

\footnotetext{
${ }^{1}$ Laboratory of Immune Regulation, Graduate School of Medicine, Osaka University, Suita, Osaka, Japan. ${ }^{2}$ WPI Immunology Frontier Research Center, Osaka University, Suita, Osaka, Japan. ${ }^{3}$ Precursory Research for Embryonic Science and Technology (PREST), Japan Science and Technology Agency, Saitama, Japan.

Correspondence: K Takeda (ktakeda@ongene.med.osaka-u.ac.jp)

Received 31 December 2008; accepted 30 January 2009; published online 4 March 2009. doi:10.1038/mi.2009.8
} 
Several molecules that inhibit activation of the TLR signaling pathways have been identified. ${ }^{23}$ TLR possesses the extracellular domain characterized by the presence of a leucine-rich repeat responsible for the microbial recognition and the intracellular Toll/IL-1 receptor (TIR) domain, and thus constitutes a large TLR/IL-1R superfamily. The single immunoglobulin (Ig) IL-1 receptor-related molecule (SIGIRR, also known as TIR8) represents a unique subgroup of the TLR/IL-1R superfamily, consisting of an extracellular single Ig domain and an intracellular TIR domain. ${ }^{24}$ SIGIRR is expressed in dendritic cells and intestinal epithelial cells, and inhibits TLR signaling. ${ }^{24,25}$ Indeed, mice lacking Sigirr show high susceptibility to intestinal inflammation induced by dextran sodium sulfate (DSS) and increased colitis-associated tumor incidence. ${ }^{26}$ Intestinal epithelial cells from Sigirr-deficient mice show increased commensal bacteriadependent cytokine production. Intestinal epithelium-specific expression of SIGIRR reduces the sensitivity to DSS-induced intestinal inflammation and tumorigenesis in Sigirr-deficient mice. These findings suggest the crucial role of SIGIRR in maintenance of intestinal homeostasis through negative regulation of TLR signaling in the intestinal mucosa. The inhibitory effect of SIGIRR is shown to be exerted through ligand-dependent interaction with TLR and the signaling molecule tumor necrosis factor (TNF)-receptor-associated factor 6 (TRAF6). ${ }^{24}$

The IL-1R-associated kinase (IRAK) family of kinases consists of four members, namely IRAK1, IRAK2, IRAK-M, and IRAK4. IRAK1, 2, and 4 are shown to mediate the TLR signaling by bridging TIR domain-containing adaptors to TRAF6 ${ }^{27}$ In contrast to other IRAK family members that are ubiquitously expressed, IRAK-M expression is limited to monocytes; it negatively regulates the TLR signaling by inhibiting formation of the IRAK1TRAF6 complex. ${ }^{28}$ Irak3 (the gene encoding IRAK-M)-deficient mice show severe inflammatory response in the intestine after infection with Salmonella typhimurium, ${ }^{28}$ indicating its role in the modulation of TLR-dependent intestinal inflammatory responses. Although there is no evidence suggesting an association between IBDs and single nucleotide polymorphisms in IRAK-M, ${ }^{29}$ further studies are needed to determine whether an impaired negative regulation of the TLR signaling pathway by IRAK-M might be partly responsible for the development of IBD.

A20 is a ubiquitin-modifying enzyme that is rapidly induced by TLR or TNF- $\alpha$ stimulation and terminates TLR-dependent responses by removing ubiquitin moieties from the signaling molecule TRAF6. ${ }^{30}$ Tnfaip 3 (the gene encoding A20)-deficient mice exhibit severe inflammation of multiple organs, including the intestine. Spontaneous inflammation in Tnfaip3-deficient mice is rescued by the introduction of the deficiency of MyD88. In addition, depletion of intestinal bacteria by treatment with broad-spectrum antibiotics diminishes the inflammation in Tnfaip3-deficient mice. ${ }^{31}$ Thus, A20 is a key negative regulator of intestinal bacteria- and TLR-dependent inflammation.

The suppressor of cytokine signaling-1 (SOCS1) was identified as a potent negative regulator of signaling pathway of various cytokines, including interferon- $\gamma$ and IL- $4 .{ }^{32}$ SOCS1 has subsequently been shown to inhibit the TLR-induced inflammatory responses. Although early lethality owing to severe inflammation of multiple organs in Socs $1^{-1-}$ mice can be prevented by the blockade of interferon- $\gamma$ signaling, macrophages from these mice still show increased cytokine production in response to TLR stimulation. ${ }^{33,34}$ The Socs 1 deficiency exacerbates the severity of colitis in mice lacking a T-cell receptor chain or DSS-treated mice presumably owing to excess TLR signaling. ${ }^{35,36}$ However, severe colitis does not develop when these mutant mice are kept in a germ-free environment. ${ }^{32}$ Thus, SOCS1 is also a key negative regulator to prevent excessive intestinal bacteria- and/or TLR-dependent inflammatory responses in the intestine.

TLR-mediated immune response can be divided into two phases, early and late. During the early phase, nuclear factor$\kappa \mathrm{B}(\mathrm{NF}-\kappa \mathrm{B})$ is swiftly recruited to constitutively and immediately accessible promoters of genes, such as TNF- $\alpha$, CXCL2, and CXCL1 (also known as KC). In the later phase of the TLR response, genes for which induction requires stimulus-dependent chromatin modifications become accessible to NF- $\kappa \mathrm{B}$ and other transcription factors, and are strongly induced. It can be noted, many of the late inducible genes, such as IL-6, IL-12p40, and IL-18, are known to be involved in the activation of T cells. It has been shown that the induction of late inducible genes is regulated, at least in part, by early inducible genes, which

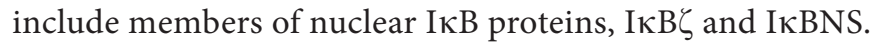
The induced $\mathrm{I} \kappa \mathrm{B} \zeta$ interacts with p50 subunit of NF- $\kappa \mathrm{B}$ and enhances the induction of late inducible genes through trimethylation of histone $\mathrm{H} 3 \mathrm{~K} 4$ in their promoters. ${ }^{37,38}$ On the other hand, IкBNS is selectively expressed in the lamina propria macrophages during early phase of TLR signaling. ${ }^{13}$ In

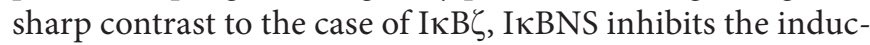
tion of the late inducible genes. ${ }^{39}$ The Nfkbid (the gene eocodes IкBNS)-deficient mice are highly susceptible to intestinal inflammation, such as DSS-induced colitis. ${ }^{39}$ Thus, the selective expression of IкBNS may provide a mechanistic basis for the hypo-responsiveness of intestinal lamina propria macrophages to TLR stimulation.

Peroxisome proliferator-activated receptor- $\gamma(\operatorname{PPAR} \gamma)$, a member of the nuclear receptor family, is highly expressed in the intestinal epithelial cells; ${ }^{40}$ it has been shown to suppress the expression of a subset of TLR-dependent genes. ${ }^{41}$ Bacteroides thetaiotaomicron, a prevalent commensal anaerobic bacterium of the human intestine, attenuates inflammatory responses by limiting duration of NF- $\kappa \mathrm{B}$ activity. PPAR $\gamma$ has been shown to be responsible for $B$. thetaiotaomicron-dependent nuclear export of RelA subunit of the NF- $\kappa B$ in intestinal epithelial cells. ${ }^{42}$ Thus, PPAR $\gamma$ is also proposed as a therapeutic target of IBDs. ${ }^{43}$

\section{TLR SIGNALING REQUIRED FOR INTESTINAL HOMEOSTASIS}

So far, we have discussed that when TLR-dependent activation of innate immunity is in excess, intestinal inflammation ensues. However, a growing body of evidence indicates that TLR-dependent recognition of commensal bacteria is required for the maintenance of the intestinal homeostasis. Mice lacking TLR signaling components (TLR2, TLR4, or MyD88) are highly susceptible to DSS-induced intestinal inflammation. ${ }^{44,45}$ 


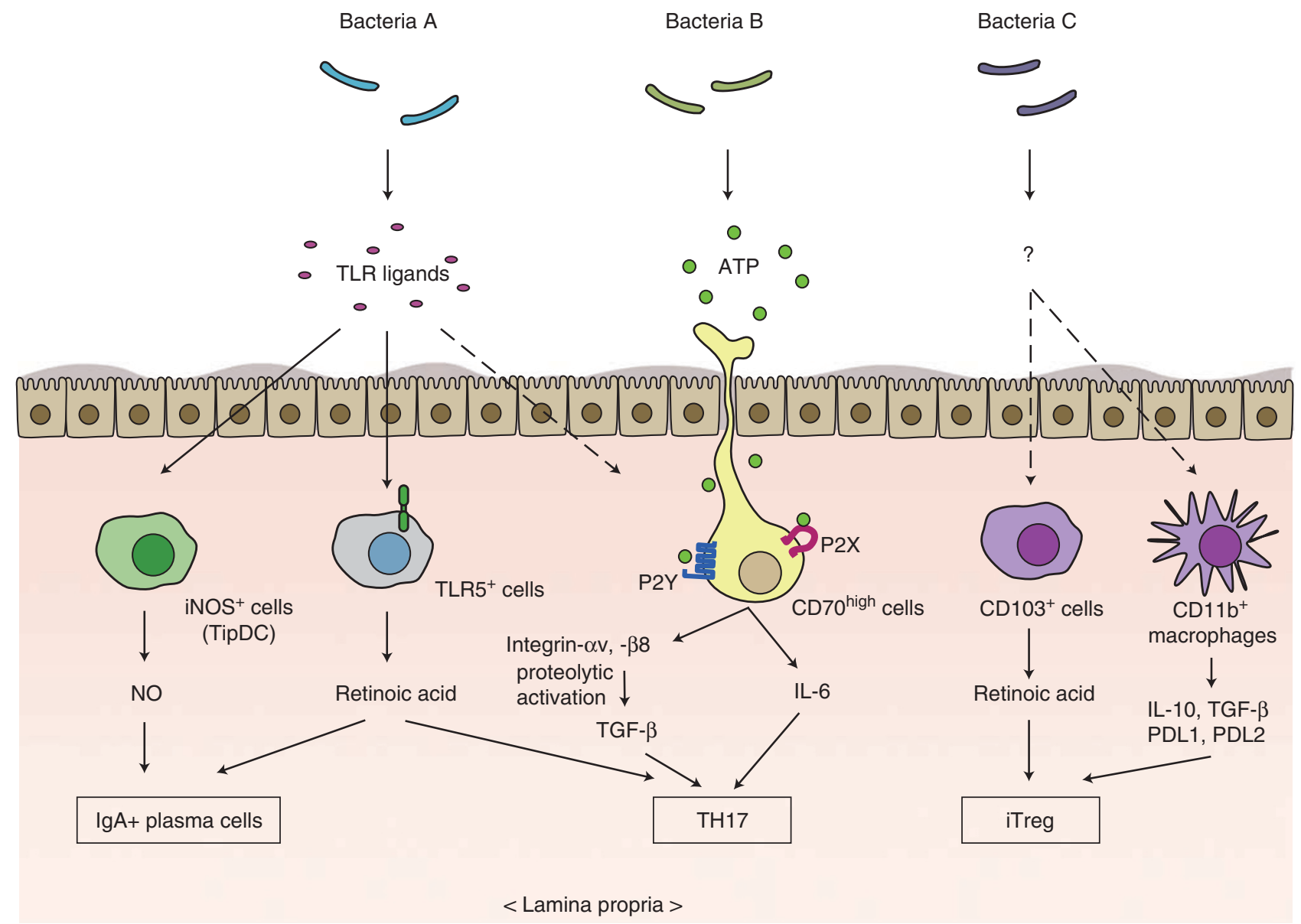

Figure $1 \mathrm{~A}$ hypothetical model for intestinal bacteria-mediated differentiation of adaptive immune cells in the lamina propria. The intestinal microflora is composed of a variety of bacteria with different characters; each of them produces stimulatory factors for host immune system. A subset of commensal bacteria (Bacteria A) produces TLR ligands, which activate specific subsets of lamina propria APCs, TipDCs, and TLR5 ${ }^{+}$DCs, thereby inducing the differentiation of $\lg \mathrm{A}^{+}$plasma cells and TH17 cells. Another subset of intestinal bacteria (Bacteria B) produces and secretes high amounts of extracellular ATP. CD70 high CX3CR1 ${ }^{+}$dendritic cells extend their dendrites into the lumen, sense the extracellular ATP through P2X and P2Y receptors, and produce IL- 6 and TGF- $\beta$-activating enzymes to promote differentiation of TH17 cells. A subset of intestinal commensal bacteria (Bacteria C) produce an as-yet unknown factor that activate CD103+ DCs or CD11 b+ macrophages to produce retinoic acid and other factors to induce differentiation of $\mathrm{iT}_{\text {regs }}$. TLR, Toll-like receptor; APC, antigen-presenting cell; IgA, immunoglobulin A; TipDC, TNF- $\alpha /$ iNOS-producing dendritic cell subset; IL-6, interleukin-6; TGF- $\beta$, transforming growth factor- $\beta$; DC, dendritic cell; $\mathrm{iT}_{\text {reg }}$, inducible $\mathrm{T}_{\text {reg }}$.

Moreover, treatment of broad-spectrum antibiotics also result in high susceptibility to DSS-induced intestinal inflammation. ${ }^{44}$ This is revealed owing to the defective expression of tissueprotective factors in the intestinal epithelial cells or defective TLR-dependent control of tight junction-associated intestinal epithelial barrier integrity. ${ }^{44,46}$ TLR9-dependent signal in intestinal epithelial cells has also been shown to maintain the intestinal homeostasis. ${ }^{47}$ Thus, these studies show that TLR-dependent recognition of commensal bacteria under normal steady-state conditions is crucial for the maintenance of intestinal homeostasis. The importance of the interaction of TLRs and commensal bacteria is also shown in other studies. Colonization of germ-free mice with $B$. thetaiotaomicron induces epithelial expression of genes involved in several intestinal functions, including nutrient absorption, mucosal barrier fortification, angiogenesis, and postnatal intestinal maturation. ${ }^{48}$ In addition, systemic immunological defect as evidenced by the imbalance of TH1/TH2 cell populations in germ-free mice is recovered by colonization with a commensal bacterium Bacteroides fragilis. A polysaccharide of $B$. fragilis is sufficient for the proper development of systemic immunity. ${ }^{49}$ Modulation of TH1/TH2 balance by the polysaccharide of $B$. fragilis is further shown to be dependent on TLR2. ${ }^{50}$ Thus, commensal bacteria-derived factors critically modulate intestinal immune responses, at least in part, through TLRs.

Another interesting aspect of TLR signaling in the intestinal mucosa is the finding that TLR-dependent signaling can induce intestinal tumorigenesis under certain circumstances. ${ }^{51}$ Thus, TLRs at the intestinal mucosal surfaces have a variety of functions, such as induction of inflammation, maintenance of homeostasis, and suppression of tumorigenesis. Further investigation is needed to learn how these yin and yang properties of TLRs are regulated in the intestine.

\section{NLR-DEPENDENT RESPONSES INTHE INTESTINE}

There are also TLR-independent mechanisms for microbial recognition by cytoplasmic molecules, including nucleotidebinding and oligomerization domain (NOD)-like receptors (NLRs), ${ }^{52-54}$ which are a large family comprising over 20 
members of proteins. ${ }^{55}$ NLRs have been implicated in the recognition of several bacterial components as well as of environmental danger signals, such as adenosine $5^{\prime}$-triphosphate (ATP), urate crystal, and silica. ${ }^{56}$ Among these NLR family members, NOD1 and NOD2 have been characterized to be involved in immune responses at the intestinal mucosa. ${ }^{53}$

Involvement of NOD proteins in the intestinal inflammation was first shown in studies in which NOD2 gene mutation associates with increased susceptibility to Crohn's disease in Western (but not Asian) populations. ${ }^{57,58}$ The mutations are located in the C-terminal leucine-rich repeat region responsible for ligand recognition. Although in vitro assays suggest that the NOD2 mutations result in a loss of function, ${ }^{59}$ whether these mutations predispose to Crohn's disease in vivo through a loss or gain of function remains a matter of debate. NOD2 is expressed in Paneth cells that are a critical source of various antimicrobial peptides. ${ }^{60}$ Nod2-deficient mice are susceptible to oral infection of Listeria monocytogenes, with defective expression of anti-microbial peptides in the small intestine. ${ }^{61}$ Furthermore, the expression of the antimicrobial peptides $\alpha$-defensins has been shown to be reduced in Crohn's disease patients with the NOD2 mutation. ${ }^{62}$ Thus, these results support the notion that the Crohn's disease-associated NOD2 mutations render the molecule hypo-responsive to the NOD2 ligands. Another study using mice carrying the NOD2 mutation that corresponds to the Crohn's diseases-associated NOD2 mutation shows that this type of the NOD2 mutation results in increased production of IL-1 $\beta$, leading to the development of intestinal inflammation. ${ }^{63}$ This study discusses that this type of mutation in mice, unlike that in humans, causes hyper-responsiveness to NOD2 stimulation. ${ }^{59}$ Another mechanism in which NOD2 is a negative regulator of TLR2 signaling is also proposed. ${ }^{64}$ In this mechanism, TLR2dependent production of IL- 12 by dendritic cells is increased in the absence of NOD2-mediated suppression, leading to aberrant TH1 cell development, which causes intestinal inflammation. ${ }^{65}$ In any case, NOD2 mutation presumably results in a marked change in the components of intestinal microflora, which play a critical role in promoting disease pathogenesis.

Involvement of NOD1, expressed in the intestinal epithelial cells, in the intestinal immune responses has also been shown. Nod1-deficient mice are highly susceptible to infection with Helicobacter pylori, indicating that NOD1 is critical to the innate immune response in the intestinal epithelia to bacterial infection. ${ }^{66}$ In addition, NOD1 gene polymorphisms are shown to be associated with susceptibility to inflammatory diseases such as Crohn's diseases and ulcerative colitis. ${ }^{67}$ Thus, NOD1 and NOD2 are both critical constituents of innate immune system in the intestinal mucosa.

\section{REGULATION OF ADAPTIVE IMMUNE SYSTEM BY COMMENSAL BACTERIA}

The intestinal mucosa also has a unique and extremely complicated immune system composed of a variety of adaptive immune cell populations, including IgA-producing plasma cells, $\gamma \delta \mathrm{T}$ cells, and $\mathrm{CD} 4^{+} \mathrm{T}$ cells dominated by a TH1 or a TH2 phenotype. In addition, recent studies have revealed that $\mathrm{CD} 4^{+}$
T cells in the intestinal mucosa comprise significant numbers of IL-17-producing cells (TH17 cells) and regulatory T cells $\left(\mathrm{T}_{\text {reg }}\right.$ cells). Furthermore, very recent reports showed the presence of IL-22-producing natural killer-like cells (NK-22 cells) in the intestinal mucosa, which are positive for NK cell markers but do not have killing activity of MHC class I-negative cells. All of these cells are particularly abundant in the intestinal mucosa and are present even at steady state. The mechanism of how the intestinal mucosa can accomplish the equipment of diverse sets of adaptive immune cell populations is not fully understood. The most commonly accepted view is that such a large variety occurs as a result of interaction between commensal bacteria, each with a distinct character, and antigen-presenting cell (APC) populations, each has a different functional bias (Figure 1). We discuss below how commensal bacteria and APCs provide a particular environment for the unique and well-balanced development of adaptive immune cells.

\section{DEVELOPMENT OF IGA-PRODUCING CELLS}

Among the adaptive immune cell populations in the intestinal mucosa, the best-characterized cells are the IgA-producing plasma cells. Normal intestinal mucosa contains abundant IgA-secreting plasma cells, and the secreted IgA plays a critical role in the host defense against pathogenic bacteria. More importantly, IgA regulates the ecological balance of commensal bacteria; in other words, IgA regulates the composition and character of intestinal microflora. For example, the lack of activation-induced cytidine deaminase (AID), which results in the defect of class-switch recombination, and thereby lack of IgA-producing plasma cell in the intestine, leads to excessive anaerobic expansion in the small intestine, particularly the segmented filamentous bacteria (SFB) ${ }^{68}$ Conversely, the addition of IgA rescues the aberrant $\mathrm{SFB}$ expansion in the small intestine of AID $^{-1-}$ mice. ${ }^{68}$ Furthermore, it has been shown that IgA does not affect the growth rate of $B$. thetaiotaomicron, but affects the expression of bacterial molecules. ${ }^{69}$ As a result, in the absence of $\operatorname{IgA}, B$. thetaiotaomicron, which is otherwise commensal to the host, elicits a more robust innate immune response, including induction of inducible nitric oxide synthase (iNOS) ${ }^{69}$ Thus, mucosal IgA can determine the nature of bacteria and is required for keeping commensal bacteria to be "commensal" to the host.

The development of IgA-secreting cells is regulated, in turn, by the presence of intestinal microflora. The germ-free mice exhibit severe reductions in their fecal IgA levels and in the numbers of lamina propria IgA-positive cells. ${ }^{5,70-72}$ The specific pathogen-free (SPF) mice treated with antibiotics, such as vancomycin and metronidazole, show similar reductions of the levels of IgA to those in germ-free mice. Thus, commensal bacteria play a critical role in the provision of a particular environment for the development of IgA-producing cells. Using gnotobiotic mice where only certain known strains of bacteria are present, it has been shown that a set of bacterial species, such as SFB and clostridia, specifically stimulate the development of IgA-producing cells. ${ }^{71,72}$ IgA concentrations of ileal contents or feces are much higher in SFB- or clostridia-monoassociated 
mice than in mice associated with whole intestinal flora (conventionalized mice). ${ }^{73}$ Thus, certain bacterial species promote differentiation of IgA-producing cells, whereas others do not or may inhibit it.

In conventional views, IgA-positive B cells are mainly generated in a $\mathrm{T}$ cell- and CD40 ligand (CD40L)-dependent manner in the germinal centers of organized follicular structures of Peyer's patches (PPs), where antigens of commensal bacteria are taken up by $\mathrm{M}$ cells located in the follicle-associated epithelium of PPs. After generation in the PPs, IgA ${ }^{+} \mathrm{B}$ cells migrate into the draining mesenteric lymph nodes, where they differentiate into IgAsecreting cells, which then home to the intestinal lamina propria. In contrast to this T-cell- and PP-dependent class-switch model, an emerging model is that a large proportion of the intestinal IgA is locally induced in the intestinal lamina propria by local factors in a T-cell-independent manner. ${ }^{74}$ Commensal bacteria are directly taken up by epithelial cells through $\mathrm{Fc}$ receptor Rn or by dendritic cells at mucosal sites. ${ }^{75,76}$ In parallel, commensal bacteria provide molecular patterns that activate TLR or other innate immune receptors in lamina propria cells. Microbial TLR ligands, such as lipopolysaccharide (LPS), can substitute for CD40L and act directly on B cells along with transforming growth factor- $\beta$ (TGF- $\beta$ ) to initiate IgA class switching. ${ }^{77}$ In addition to engaging TLRs on $B$ cells, microbial products stimulate the release of B-cell-activating factor (BAFF; also known as BLyS) and a proliferation-inducing ligand (APRIL) by dendritic cells. BAFF and APRIL are functionally related to CD40L and promote AID expression and IgA class switching in a CD40Lindependent manner. Recently, it has been shown that BAFF and APRIL are co-expressed with iNOS in CD11 $\mathrm{c}^{\text {lo }}$ dendritic cells in the intestinal lamina propria. ${ }^{78} \mathrm{The}_{\mathrm{NOS}}{ }^{+}$dendritic cells express gaseous nitric oxide, which induces BAFF and APRIL by an unknown mechanism. The iNOS ${ }^{+}$dendritic cells are also positive for TNF- $\alpha$; thus, they are called as a naturally occurring TNF- $\alpha /$ iNOS-producing dendritic cell subset (TipDC). As the number of TipDC is much reduced in the lamina propria of

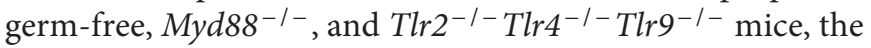
expression of iNOS in TipDC is mediated by microbial products and signaling through TLR. ${ }^{78}$ It is likely that TipDCs are "IgA-inducing DCs" that recognize specific species of commensal bacteria and preferentially promote B-cell differentiation into IgA-producing cells in the intestinal lamina propria in situ (Figure 1).

In the context of lamina propria dendritic cells that preferentially mediate $\operatorname{IgA}^{+}$cell differentiation, there is another subset of lamina propria dendritic cells in the small intestine, that is, TLR $5{ }^{+} \mathrm{CD} 11 \mathrm{c}^{\mathrm{hi}} \mathrm{CD} 11 \mathrm{bi}$ cells that mediate $\mathrm{IgA}{ }^{+}$cell differentiation through the production of retinoic acid. ${ }^{79}$ Upon stimulation by a TLR 5 ligand flagellin, TLR $5^{+}$dendritic cells express retinal dehydrogenase 2 (Raldh2), which enzymatically converts vitamin A into retinoic acid. Retinoic acid then acts on naive B cells to differentiate into IgA-producing plasma cells (Figure 1). As $\operatorname{Tlr}^{-1-}$ mice still have normal numbers of IgA ${ }^{+}$cells in the intestinal lamina propria, this mechanism is not essential during steady state. However, TLR $5^{+}$dendritic cells might play a critical role during infections by pathogenic bacteria, because flagella are usually observed in pathogenic bacteria, but are rarely seen in intestinal commensal bacteria. Further investigation is required to decipher the relationship between TipDC and $\mathrm{TLR}^{+}{ }^{+}$dendritic cells for IgA class switching during health and disease.

Recently, much attention has been focused on isolated lymphoid follicles (ILFs) in the lamina propria as the site where T-cell-independent induction of IgA class switching of B cells takes place. ${ }^{80}$ It was shown that although $T c r b^{-1-} \mathrm{Tcrd}^{-1-}$ mice fail to generate $\operatorname{IgA}^{+} \mathrm{B}$ cells within PP follicles, they have an almost normal level of luminal IgA. ${ }^{80}$ Instead of PP, a considerable number of IgA ${ }^{+}$plasma cells can be observed in the lamina propria of the small intestine, particularly in ILFs. ${ }^{80}$ These facts support the idea that the T-cell-independent development of IgA ${ }^{+}$ cells predominantly takes place in ILF. Interestingly, the formation of ILFs is dependent on the presence of commensal bacteria, as is evident by the analysis of germ-free mice in which ILF is defective. ${ }^{81}$ To induce the genesis of ILFs, peptidoglycan from Gram-negative commensal bacteria, such as Bacteroides genus, is required. Peptidoglycan is recognized by the NOD1 receptor in epithelial cells, resulting in the induction of chemokines and recruitment of cells required for the formation of ILFs. Conversely, in the absence of ILFs, the composition of the intestinal bacterial community is profoundly altered: Clostridiales and Bacteroides markedly expand, whereas the Gram-negative Lactobacillaceae are reduced in ILF-deficient mice. ${ }^{81}$ Thus, it is highly likely that the steady and well-balanced state of the intestinal mucosa is achieved by a dynamic equilibrium between immune system, including ILF-IgA system, and intestinal microflora.

\section{DEVELOPMENT OFTH17 CELLS}

TH17 cells constitute a subset of activated $\mathrm{CD} 4{ }^{+} \mathrm{T}$ cells that are characterized by the production of IL-17A, IL-17F, IL-21, and IL-22. The produced IL-17 acts on a broad range of immune and non-immune cells and regulates granulopoiesis, neutrophil recruitment, and induction of antimicrobial peptides. IL-22 acts on epithelial cells of the gastrointestinal tract and skin (but not on immune cells that are defective of IL-22 receptor expression) and leads to the upregulation of antimicrobial proteins and cellular proliferation. By producing these cytokines, TH17 cells play critical roles in the regulation of host defense against a variety of infections by fungus and bacteria, such as Klebsiella pneumoniae, Streptococcus pneumoniae, and Citrobacter rodentium..$^{82-84}$ However, at the same time, the aberrant TH17 response can lead to severe autoimmune diseases, such as multiple sclerosis, rheumatoid arthritis, and IBDs. Contrary to their inflammatory roles, IL-17 and IL-22 also have a protective role in inflammation. For example, it has been shown that IL-22 counteracts the destructive nature of inflammation during hepatitis: Il22deficient mice are highly sensitive to ConA-induced acute liver inflammation. ${ }^{85}$ Thus, depending on the circumstances, TH17 cells may serve as an inflammatory or a protective population to maintain tissue homeostasis.

Specific microbial stimuli detected by innate immune cells initiate TH17 differentiation, where a "master regulator" transcription factor ROR $\gamma \mathrm{t}$ is induced by the local cytokine milieu, including 
IL- 6 and TGF- $\beta$ in the mouse system. ${ }^{86}$ It should be noted that all the TH17 cells are not the same and different environments induce TH17 cells with different characteristics. ${ }^{87}$ For example, the presence of ligands for aryl hydrocarbon receptor affects the expression of IL-22, but not other TH17 cytokines. ${ }^{88}$ Furthermore, although TGF- $\beta$ and IL- 6 are essential for the "initiation" of TH17 differentiation, ${ }^{84}$ they are not sufficient to induce fully inflammatory TH17 cells. During stimulation with TGF- $\beta$ and IL6, TH17 cells express IL-23 receptor and become responsive to IL-23. ${ }^{89}$ In the presence of the pro-inflammatory cytokine IL-23, TH17 cells can expand and mediate a full-blown TH17 response. ${ }^{87,89}$ Indeed, despite the fact that the development of TH17 cells in C. rodentium-infected mice is promoted by the TGF- $\beta$-IL- 6 pathway, IL-23 is indispensable in these mice for the TH17 response that protects against $C$. rodentium-driven colitis. ${ }^{84}$ In the presence of excess amounts of IL-23, TH17 cells become pathogenic and induce autoimmune disease in mice. Indeed, using a T-cell transfer model of experimental allergic encephalomyelitis, a recent study showed that $\mathrm{TH} 17$ cells that are expanded in the presence of IL-23, but not with TGF- $\beta$ plus IL-6, induce disease in mice. ${ }^{90} \mathrm{IL}-23$ is also shown to mediate intestinal inflammation. ${ }^{91,92}$ Moreover, in humans, it has been shown that variants of the IL23R gene are linked to susceptibility to IBDs. ${ }^{93}$

Importantly, the intestinal lamina propria is a unique site for the presence of TH17 cells in healthy animals. TH17 cells are present at high frequencies in the small and large intestinal lamina propria but not in the spleen, mesenteric lymph nodes, or PPs of healthy mice housed in a SPF environment. ${ }^{86,94,95}$ In the small and large intestines, about $10 \%$ of $\mathrm{CD} 4{ }^{+}$cells are IL- $17^{+}$ cells; and $80-90 \%$ of the IL- $17^{+}$cells are CD $4^{+} \mathrm{TCR} \alpha \beta^{+} \mathrm{TH} 17$ cells. ${ }^{95}$ In contrast, in extra-intestinal sites, only a very small proportion of $\mathrm{CD} 4{ }^{+} \mathrm{TCR} \alpha \beta^{+} \mathrm{T}$ cells express IL- 17 at steady state (fewer than $1 \%$ ), and $<10 \%$ of $\mathrm{IL}-17^{+}$cells are CD $4^{+}$cells (NK cells, CD ${ }^{+}$T cells, and TCR $\gamma \delta^{+} \mathrm{T}$ cells also express IL-17 and ROR $\gamma \mathrm{t}$ in extra-intestinal sites). ${ }^{95}$ The number of TH17 cells in the intestinal lamina propria increases with age, correlating with the development of intestinal microflora. ${ }^{94}$ Furthermore, in germ-free mice or antibiotics-treated mice, the numbers of lamina propria TH17 cell are greatly reduced. ${ }^{94,95}$ Therefore, similar to the case of IgA-producing plasma cells, the development of TH17 cells is dependent on the stimulation by intestinal commensal bacteria. Interestingly, mice obtained from different commercial vendors have marked differences in the number of TH17 cells in their lamina propria: C57BL/6 mice from the SPF facilities of Taconic Farms (Hudson, NY) have higher numbers of TH17 cells than those from the Jackson Laboratory (Bar Harbor, ME). ${ }^{95}$ The difference in the presence of intestinal TH17 cells is correlated with the presence of members of the cytophaga-flavobacter-bacteroides phylum. ${ }^{95}$ Thus, it is likely that TH17 cells are induced in the lamina propria in response to specific components of the commensal microflora, which presumably activate innate signaling pathways in subsets of APCs in the lamina propria. In sharp contrast to this notion, a recent report showed that commensal bacteria may inhibit TH17 differentiation through IL-25. ${ }^{96}$ Further investigative studies will need to clarify these discrepant controversies.
Two groups independently showed that Myd88 and Trif doubly deficient mice had normal numbers of lamina propria TH17 cells in the small and large intestines, and concluded that the TH17 development in the lamina propria is independent of TLR signaling at least at steady state. ${ }^{94,95}$ However, a possible caveat to this interpretation is that these mutant mice might have altered intestinal microflora. Indeed, it was shown that Myd88 deficiency changes the composition of the distal gut microbiota: $M y d 88^{-1-}$ mice have a significantly lower Firmicutes/Bacteroidetes ratio compared with $M y d 88^{+/-}$mice. ${ }^{97}$ Furthermore, another group showed that Tlr9-deficient mice have decreased numbers of lamina propria TH17 cells. ${ }^{98}$ Thus, to formally conclude the involvement of TLR signaling in TH17 differentiation, gnotobiotic studies using germ-free mice deficient for MyD88 or TLRs colonized with a certain TH17-inducing bacteria needs to be carried out.

Besides TLR ligands, bacteria have been shown to generate and secrete large amounts of extracellular ATP. ${ }^{99}$ ATP is a well-known source of intracellular energy transfer; however, it also serves essential roles in extracellular signaling processes. Extracellular ATP binds and activates the cell-surface ionotrophic (P2X) and metabotropic (P2Y) purinergic receptors, which deliver intracellular signals through ion channels or G-proteins, respectively. ${ }^{100,101}$ In the context of the immune system, extracellular ATP is known to modulate immune cell functions, such as phagocytosis, chemotaxis, and cytokine production. ${ }^{100,101}$ Particularly, the induction of ATP signaling through $\mathrm{P} 2 \mathrm{X} 7$ receptor is known to be involved in the activation of caspase- 1 through the assembly of a cytosolic protein complex that is known as the "inflammasome" and required for the conversion of immature form of IL- $1 \beta$ into the mature form. ${ }^{102}$ ATP can be quickly degraded in the extracellular space by ATPases into ADP, AMP, or adenosine forms. However, intestinal commensal bacteria seem to produce high amounts of ATP beyond the capacity of host ATPases. Indeed, ATP concentrations in intestinal luminal contents of SPF mice are very high, but found at very low levels in germ-free mice or antibiotics-treated mice. ${ }^{94}$ Moreover, high ATP concentration can be detected in the supernatant of in vitro-cultured intestinal commensal bacteria. The addition of the culture supernatant of intestinal commensal bacteria markedly enhances differentiation of IL-17-expressing cells, and this TH17 differentiation is severely inhibited by the presence of ATP-degrading enzyme, apyrase. ${ }^{94}$ Furthermore, treatment of germ-free mice with ATP markedly increases the numbers of IL-17-producing CD $4{ }^{+}$cells. ${ }^{94}$ Thus, extracellular ATP is one of the critical TH17-inducing factors produced by intestinal commensal bacteria (Figure 1).

Lamina propria CD $11 c^{+}$cells, particularly CD70high cells, express high levels of $\mathrm{P} 2 \mathrm{X}$ and $\mathrm{P} 2 \mathrm{Y}$ receptors and also express several genes, including IL-6, IL-23, integrin- $\alpha \mathrm{V}$, and integrin$\beta 8$, in response to ATP stimulation. ${ }^{94}$ Integrin- $\alpha \mathrm{V}$ and integrin$\beta 8$ are involved in the conversion of latent form of TGF- $\beta$ into the active form through triggering degradation of latency-associated protein binding to TGF- $\beta .^{103} \mathrm{CD} 70^{\text {high }} \mathrm{CD} 11 \mathrm{c}^{+}$cells preferentially induce the differentiation of naive $\mathrm{CD} 4^{+} \mathrm{T}$ cells into TH17 cells. ${ }^{94}$ There is another report showing that lamina 
propria $\mathrm{CD} 11 \mathrm{~b}^{\text {high }} \mathrm{CD} 11 \mathrm{c}^{+}$cells are a TH17-inducing dendritic cell population. ${ }^{104}$ Both $\mathrm{CD} 70^{\text {high }}$ and $\mathrm{CD} 11 \mathrm{~b}^{\text {high }}$ populations express CX3CR1, a chemokine receptor mediates the extension of cellular dendrites of dendritic cells between the tight junctions of epithelial cells to take up luminal bacteria. ${ }^{76}$ Thus, it is likely that $C D 70^{\text {high }}$ and $C D 11 b^{\text {high }}$ populations seem to be, at least partially, overlapping with each other and both play a critical role in the TH17 differentiation in the lamina propria in situ.

Collectively, the current model for TH17 differentiation in the intestinal lamina propria therefore involves production of high amounts of ATP or other TH17-inducing molecules by a specific subset of intestinal bacteria, followed by the activation of a specific subset of lamina propria dendritic cells expressing CD70 or $\mathrm{CD} 11 \mathrm{~b}$ and the production of IL-6, IL-23, and TGF- $\beta$ (Figure 1). In inflammatory conditions, the differentiation of intestinal TH17 cells seems to be enhanced by TLR-dependent pathway, as flagellin activates TLR ${ }^{+}$lamina propria dendritic cells to induce TH17 cell differentiation..$^{79}$ It will be important to understand further the specific types of TH17-inducing intestinal bacteria as well as of TH17-inducing APC subsets during steady and disease states.

\section{DEVELOPMENT OF INDUCIBLE $T_{\text {REGS }}$ CELLS}

$\mathrm{T}_{\text {regs }}$ play an indispensable role in maintaining immunological unresponsiveness to self-antigens and in suppressing excessive immune responses deleterious to the host. ${ }^{105} \mathrm{~T}_{\text {regs }}$ express the transcription factor fork-head box P3 (Foxp3), which is critical to initiating a genetic program for the suppressive functions of $\mathrm{T}_{\text {regs }}$. Besides evidence that natural Foxp $3^{+} \mathrm{T}_{\text {reg }}$ arise and mature in the thymus, there is mounting evidence that Foxp $3^{+} \mathrm{T}_{\text {reg }}$ can develop extra-thymically under certain conditions, particularly in the intestinal mucosa. Indeed, the peripheral conversion of Foxp $3^{-} \mathrm{CD} 4^{+} \mathrm{T}$ cells into Foxp $3^{+} \mathrm{T}_{\text {regs }}$ can be observed experimentally in the intestinal lamina propria and gut-associated lymphoid tissue (GALT) after oral exposure to antigen. ${ }^{106,107}$ These inducible $\mathrm{T}_{\text {reg }}\left(\mathrm{iT}_{\text {regs }}\right)$ are believed to mediate peripheral $\mathrm{T}$ cell tolerance to antigens derived from commensal flora or of dietary origin. Furthermore, a high percentage of $\mathrm{T}$ cells in the small and large intestines spontaneously produce anti-inflammatory cytokine IL-10. ${ }^{108} \mathrm{IL}-10$ is not expressed by thymic natural $\mathrm{T}_{\text {regs }}$, but can be detectable in lamina propria Foxp $3^{+} \mathrm{CD} 4^{+} \mathrm{T}$ cells. As $\mathrm{T}_{\text {reg }}$-specific disruption of IL-10 in mice (Foxp3-Cre $\times I L-10^{\text {flox/flox }}$ ) results in severe autoimmune colitis, ${ }^{109} \mathrm{~T}_{\text {regs }}$ are the main source of intestinal IL-10, which play a critical role in maintenance of intestinal immune homeostasis.

Several subsets of dendritic cells with regulatory properties have been described with the capacity to induce $\mathrm{iT}_{\text {regs }}$ or induce oral tolerance at steady-state conditions. Particularly, there are several reports that indicate that a CD103 (also known as $\alpha \mathrm{E}$ integrin)-expressing dendritic cell population in the lamina propria preferentially promotes the generation and homing of $\mathrm{iT}_{\text {reg }}$ cells to the intestinal mucosa. ${ }^{106,107} \mathrm{CD} 103^{+}$dendritic cells express retinal dehydrogenase (RALDH) and produce high amounts of the vitamin A metabolite retinoid acid, which mediates the expression of the gut-homing receptors on T cells, and at the same time enhances $\mathrm{iT}_{\text {reg }}$ differentiation in the gut by cooperating with TGF- $\beta$ (Figure 1).
In the context of $\mathrm{iT}_{\text {reg }}$-inducing APCs in the gut, $\mathrm{CD} 11 \mathrm{~b}^{\text {high }} \mathrm{CD} 11 \mathrm{c}^{-}$lamina propria macrophages have also been shown to preferentially promote $\mathrm{iT}_{\text {regs }}$ development. ${ }^{104}$ The lamina propria macrophages have high expression of genes encoding anti-inflammatory molecules, including IL-10, TGF$\beta 1$, TGF- $\beta 3$, programmed cell death 1 ligand 1 (PDL1), PDL2. As a result, the lamina propria macrophages preferentially induce the differentiation of naive $\mathrm{CD} 4^{+} \mathrm{T}$ cells into Foxp3and IL-10-expressing cells. Collectively, the preferential induction of $\mathrm{iT}_{\text {reg }}$ cells at the mucosal site is attributed, at least in part, to the gut-specific presence of $\mathrm{CD} 103^{+}$dendritic cells and $\mathrm{CD} 11 \mathrm{~b}^{\text {high }} \mathrm{CD} 11 \mathrm{c}^{-}$macrophages, both of which have a functional bias for $\mathrm{iT}_{\text {reg }}$ induction (Figure 1).

Interestingly, it was recently reported that the presence of $B$. fragilis in the intestine suppress inflammation through the induction of IL-10-producing T cells. ${ }^{110}$ This anti-inflammatory effect by $B$. fragilis is achieved by polysaccharide $A$, which also prevents TH1 and TH17 effector T-cell-mediated inflammation caused by Helicobacter hepaticus in the intestine. On the other hand, DNA from commensal bacteria is shown to limit $\mathrm{iT}_{\text {reg }}$ development through TLR9. ${ }^{98}$ These studies suggest that the interaction between a specific subset of intestinal bacteria and a specific subset of APCs positively and negatively regulate $\mathrm{iT}_{\text {reg }}$ differentiation in the intestinal mucosa.

\section{CONCLUSION AND PERSPECTIVES}

Considerable progress has been made in improving our understanding of the roles of TLRs and NLRs in the induction of inflammation against pathogens: In isolated individual cells, their signaling and consequences look rather simple. Thus, one might envisage that this can be applied to the in vivo situation, where the activation of TLRs and NLRs only occurs when intestinal bacteria accidentally invade the mucosa and simply results in inflammation. However, we now know that the actual and entire picture of the intestinal innate immune system is not so simple. Not only pathogenic bacteria but also commensal bacteria continuously provide activation signals to TLRs and NLRs. The continuous activation of host cells, particularly epithelial cells, results in continuous production of tissue-repairing factors for maintaining intestinal homeostasis, without which intestinal barrier injury and bacterial translocation could occur. ${ }^{111}$ The innate immune system is also required for the production of antimicrobial peptides and lectins by Paneth cells, which critically control components of intestinal microflora to create a mutually beneficial relationship. ${ }^{112}$ Accordingly, the deficiency of innate immune signaling in $\mathrm{Myd} 88^{-1-}$ mice or $\mathrm{Nod1}^{-1-}$ mice for example, results in dramatic changes of the composition of gut microbiota. ${ }^{81,97,112}$ Furthermore, recent evidences suggest that innate immune signaling plays a role in oncogenesis. ${ }^{51}$ Further investigation is clearly required for the innate immune signaling for the maintenance of mucosal homeostasis in vivo.

The recent investigations also provide invaluable insight into the development and action of adaptive immune cells. In particular, recent studies have revealed the role of TH17 cell and $\mathrm{T}_{\text {reg }}$ cells in the regulation of intestinal homeostasis and have implicated these subsets in IBDs. Considering the requirement 
of TGF- $\beta$ for the differentiation of IgA-producing plasma cells, $\mathrm{TH} 17$ cell and $\mathrm{iT}_{\text {reg }}$ cell, the interaction between commensal bacteria and intestinal immune and non-immune cells may provide a specific environment rich in active form of TGF- $\beta$. It is important to study further the molecular basis for this issue. In addition, accumulating evidence suggest the presence of functionally specified dendritic cell subsets in intestinal mucosa. ${ }^{113,114}$ In the future it will be important to understand more details regarding the specific types and features of intestinal bacteria for differential adaptive immune cell differentiation and how they interact with specific intestinal APC subsets during the steady state and also inflammatory processes.

\section{ACKNOWLEDGMENTS}

This work was supported by Grants-in-Aid for Scientific Research from PRESTO, the Ministry of Education, Culture, Sports, Science and Technology, the Ministry of Health, Labour and Welfare, the Osaka Foundation for the Promotion of Clinical Immunology, the Ichiro Kanehara Foundation, Sumitomo Foundation, Senri Life Science Foundation and the Naito Foundation.

\section{DISCLOSURE}

The authors declared no conflict of interest.

(C) 2009 Society for Mucosal Immunology

\section{REFERENCES}

1. Ley, R.E., Peterson, D.A. \& Gordon, J.I. Ecological and evolutionary forces shaping microbial diversity in the human intestine. Cell 124, 837-848 (2006).

2. Savage, D.C. Microbial ecology of the gastrointestinal tract. Annu. Rev. Microbiol. 31, 107-133 (1977).

3. Hooper, L.V. \& Gordon, J.I. Commensal host-bacterial relationships in the gut. Science 292, 1115-1118 (2001).

4. Eckburg, P.B. et al. Diversity of the human intestinal microbial flora. Science 308, 1635-1638 (2005).

5. Macpherson, A.J. \& Harris, N.L. Interactions between commensal intestinal bacteria and the immune system. Nat. Rev. Immunol. 4, 478-485 (2004).

6. Backhed, F., Ley, R.E., Sonnenburg, J.L., Peterson, D.A. \& Gordon, J.I. Host-bacterial mutualism in the human intestine. Science $\mathbf{3 0 7}$, 1915-1920 (2005)

7. Strober, W., Fuss, I. \& Mannon, P. The fundamental basis of inflammatory bowel disease. J. Clin. Invest. 117, 514-521 (2007).

8. Bouma, G. \& Strober, W. The immunological and genetic basis of inflammatory bowel disease. Nat. Rev. Immunol. 3, 521-533 (2003).

9. Janeway, C.A. Jr. \& Medzhitov, R. Innate immune recognition. Annu. Rev. Immunol. 20, 197-216 (2002).

10. Takeda, K., Kaisho, T. \& Akira, S. Toll-like receptors. Annu. Rev. Immunol. 21, 335-376 (2003).

11. Ishii, K.J., Koyama, S., Nakagawa, A., Coban, C. \& Akira, S. Host innate immune receptors and beyond: making sense of microbial infections. Cell Host Microbe 3, 352-363 (2008).

12. Iwasaki, A. \& Medzhitov, R. Toll-like receptor control of the adaptive immune responses. Nat. Immunol. 5, 987-995 (2004).

13. Hirotani, T. et al. The nuclear IkappaB protein IkappaBNS selectively inhibits lipopolysaccharide-induced IL-6 production in macrophages of the colonic lamina propria. J. Immunol. 174, 3650-3657 (2005).

14. Kamada, N. et al. Abnormally differentiated subsets of intestinal macrophage play a key role in Th1-dominant chronic colitis through excess production of IL-12 and IL-23 in response to bacteria. J. Immunol. 175, 6900-6908 (2005).

15. Smythies, L.E. et al. Human intestinal macrophages display profound inflammatory anergy despite avid phagocytic and bacteriocidal activity. J. Clin. Invest. 115, 66-75 (2005).

16. Smith, P.D., Ochsenbauer-Jambor, C. \& Smythies, L.E. Intestinal macrophages: unique effector cells of the innate immune system. Immunol. Rev. 206, 149-159 (2005).
17. Takeda, K. et al. Enhanced Th1 activity and development of chronic enterocolitis in mice devoid of Stat3 in macrophages and neutrophils. Immunity 10, 39-49 (1999).

18. Kobayashi, M. et al. Toll-like receptor-dependent production of IL-12p40 causes chronic enterocolitis in myeloid cell-specific Stat3-deficient mice. J. Clin. Invest. 111, 1297-1308 (2003).

19. Rakoff-Nahoum, S., Hao, L. \& Medzhitov, R. Role of toll-like receptors in spontaneous commensal-dependent colitis. Immunity 25, 319-329 (2006).

20. Rugtveit, J. et al. Cytokine profiles differ in newly recruited and resident subsets of mucosal macrophages from inflammatory bowel disease. Gastroenterology 112, 1493-1505 (1997).

21. Schenk, M., Bouchon, A., Seibold, F. \& Mueller, C. TREM-1-expressing intestinal macrophages crucially amplify chronic inflammation in experimental colitis and inflammatory bowel diseases. J. Clin. Invest. 117, 3097-3106 (2007).

22. Kamada, N. et al. Unique CD14 intestinal macrophages contribute to the pathogenesis of Crohn disease via IL-23/IFN-gamma axis. J. Clin. Invest. 118, 2269-2280 (2008)

23. Liew, F.Y., Xu, D., Brint, E.K. \& O'Neill L, A. Negative regulation of Toll-like receptor-mediated immune responses. Nat. Rev. Immunol. 5, 446-458 (2005).

24. Wald, D. et al. SIGIRR, a negative regulator of Toll-like receptorinterleukin 1 receptor signaling. Nat. Immunol. 4, 920-927 (2003).

25. Garlanda, C. et al. Intestinal inflammation in mice deficient in Tir8, an inhibitory member of the IL-1 receptor family. Proc. Natl Acad. Sci. USA 101, 3522-3526 (2004)

26. Xiao, H. et al. The Toll-interleukin-1 receptor member SIGIRR regulates colonic epithelial homeostasis, inflammation, and tumorigenesis. Immunity 26, 461-475 (2007).

27. Kawagoe, T. et al. Sequential control of Toll-like receptor-dependent responses by IRAK1 and IRAK2. Nat. Immunol. 9, 684-691 (2008).

28. Kobayashi, K. et al. IRAK-M is a negative regulator of Toll-like receptor signaling. Cell 110, 191-202 (2002).

29. Weersma, R.K. et al. Association of interleukin-1 receptor-associated kinase M (IRAK-M) and inflammatory bowel diseases. Scand. J. Gastroenterol. 42, 827-833 (2007).

30. Boone, D.L. et al. The ubiquitin-modifying enzyme A20 is required for termination of Toll-like receptor responses. Nat. Immunol. 5, 1052-1060 (2004).

31. Turer, E.E. et al. Homeostatic MyD88-dependent signals cause lethal inflammation in the absence of A20. J. Exp. Med. 205, 451-464 (2008)

32. Yoshimura, A., Naka, T. \& Kubo, M. SOCS proteins, cytokine signalling and immune regulation. Nat. Rev. Immunol. 7, 454-465 (2007).

33. Nakagawa, R. et al. SOCS-1 participates in negative regulation of LPS responses. Immunity 17, 677-687 (2002).

34. Kinjyo, I. et al. SOCS1/JAB is a negative regulator of LPS-induced macrophage activation. Immunity 17, 583-591 (2002).

35. Chinen, T. et al. Suppressor of cytokine signaling-1 regulates inflammatory bowel disease in which both IFNgamma and IL-4 are involved. Gastroenterology 130, 373-388 (2006).

36. Horino, J. et al. Suppressor of cytokine signaling-1 ameliorates dextran sulfate sodium-induced colitis in mice. Int. Immunol. 20, 753-762 (2008).

37. Yamamoto, M. et al. Regulation of Toll//L-1-receptor-mediated gene expression by the inducible nuclear protein IkappaBzeta. Nature $\mathbf{4 3 0}$, 218-222 (2004)

38. Kayama, H. et al. Class-specific regulation of pro-inflammatory genes by MyD88 pathways and IkappaBzeta. J. Biol. Chem. 283, 12468-12477 (2008).

39. Kuwata, H. et al. IkappaBNS inhibits induction of a subset of Toll-like receptor-dependent genes and limits inflammation. Immunity 24, 41-51 (2006).

40. Dubuquoy, L. et al. Impaired expression of peroxisome proliferatoractivated receptor gamma in ulcerative colitis. Gastroenterology 124 1265-1276 (2003).

41. Ogawa, S. et al. Molecular determinants of crosstalk between nuclear receptors and toll-like receptors. Cell 122, 707-721 (2005).

42. Kelly, D. et al. Commensal anaerobic gut bacteria attenuate inflammation by regulating nuclear-cytoplasmic shuttling of PPAR-gamma and RelA. Nat. Immunol. 5, 104-112 (2004).

43. Dubuquoy, L. et al. PPARgamma as a new therapeutic target in inflammatory bowel diseases. Gut 55, 1341-1349 (2006). 
44. Rakoff-Nahoum, S., Paglino, J., Eslami-Varzaneh, F., Edberg, S. \& Medzhitov, R. Recognition of commensal microflora by toll-like receptors is required for intestinal homeostasis. Cell 118, 229-241 (2004).

45. Araki, A. et al. MyD88-deficient mice develop severe intestinal inflammation in dextran sodium sulfate colitis. J. Gastroenterol. 40, 16-23 (2005).

46. Cario, E., Gerken, G. \& Podolsky, D.K. Toll-like receptor 2 controls mucosal inflammation by regulating epithelial barrier function. Gastroenterology 132, 1359-1374 (2007).

47. Lee, J. et al. Maintenance of colonic homeostasis by distinctive apical TLR9 signalling in intestinal epithelial cells. Nat. Cell. Biol. 8, 1327-1336 (2006).

48. Hooper, L.V. et al. Molecular analysis of commensal host-microbial relationships in the intestine. Science 291, 881-884 (2001).

49. Mazmanian, S.K., Liu, C.H., Tzianabos, A.O. \& Kasper, D.L. An immunomodulatory molecule of symbiotic bacteria directs maturation of the host immune system. Cell 122, 107-118 (2005).

50. Wang, Q. et al. A bacterial carbohydrate links innate and adaptive responses through Toll-like receptor 2. J. Exp. Med. 203, 2853-2863 (2006).

51. Rakoff-Nahoum, S. \& Medzhitov, R. Regulation of spontaneous intestinal tumorigenesis through the adaptor protein MyD88. Science 317, 124-127 (2007).

52. Meylan, E., Tschopp, J. \& Karin, M. Intracellular pattern recognition receptors in the host response. Nature 442, 39-44 (2006).

53. Inohara, N., Chamaillard, M., McDonald, C. \& Nunez, G. NOD-LRR proteins: role in host-microbial interactions and inflammatory disease. Annu. Rev. Biochem. 74, 355-383 (2005).

54. Fritz, J.H., Ferrero, R.L., Philpott, D.J. \& Girardin, S.E. Nod-like proteins in immunity, inflammation and disease. Nat. Immunol. 7, 1250-1257 (2006).

55. Martinon, F. \& Tschopp, J. NLRs join TLRs as innate sensors of pathogens. Trends Immunol. 26, 447-454 (2005).

56. Petrilli, V., Dostert, C., Muruve, D.A. \& Tschopp, J. The inflammasome: a danger sensing complex triggering innate immunity. Curr. Opin. Immunol. 19, 615-622 (2007).

57. Ogura, Y. et al. A frameshift mutation in NOD2 associated with susceptibility to Crohn's disease. Nature 411, 603-606 (2001).

58. Hugot, J.P. et al. Association of NOD2 leucine-rich repeat variants with susceptibility to Crohn's disease. Nature 411, 599-603 (2001).

59. van Heel, D.A. et al. Muramyl dipeptide and toll-like receptor sensitivity in NOD2-associated Crohn's disease. Lancet 365, 1794-1796 (2005).

60. Hisamatsu, T. et al. CARD15/NOD2 functions as an antibacterial factor in human intestinal epithelial cells. Gastroenterology 124, 993-1000 (2003).

61. Kobayashi, K.S. et al. Nod2-dependent regulation of innate and adaptive immunity in the intestinal tract. Science 307, 731-734 (2005).

62. Wehkamp, J. et al. NOD2 (CARD15) mutations in Crohn's disease are associated with diminished mucosal alpha-defensin expression. Gut 53, 1658-1664 (2004).

63. Maeda, S. et al. Nod2 mutation in Crohn's disease potentiates NFkappaB activity and IL-1 beta processing. Science 307, 734-738 (2005).

64. Strober, W., Murray, P.J., Kitani, A. \& Watanabe, T. Signalling pathways and molecular interactions of NOD1 and NOD2. Nat. Rev. Immunol. 6, 9-20 (2006).

65. Watanabe, T. et al. Nucleotide binding oligomerization domain 2 deficiency leads to dysregulated TLR2 signaling and induction of antigen-specific colitis. Immunity 25, 473-485 (2006).

66. Viala, J. et al. Nod1 responds to peptidoglycan delivered by the Helicobacter pylori cag pathogenicity island. Nat. Immunol. 5, 1166-1174 (2004).

67. McGovern, D.P. et al. Association between a complex insertion/deletion polymorphism in NOD1 (CARD4) and susceptibility to inflammatory bowel disease. Hum. Mol. Genet. 14, 1245-1250 (2005).

68. Fagarasan, S. et al. Critical roles of activation-induced cytidine deaminase in the homeostasis of gut flora. Science 298, 1424-1427 (2002).

69. Peterson, D.A., McNulty, N.P., Guruge, J.L. \& Gordon, J.I. IgA response to symbiotic bacteria as a mediator of gut homeostasis. Cell Host Microbe 2, 328-339 (2007).

70. Cebra, J.J. Influences of microbiota on intestinal immune system development. Am. J. Clin. Nutr. 69, 1046S-1051S (1999).

71. Talham, G.L., Jiang, H.Q., Bos, N.A. \& Cebra, J.J. Segmented filamentous bacteria are potent stimuli of a physiologically normal state of the murine gut mucosal immune system. Infect. Immun. 67, 1992-2000 (1999).

72. Umesaki, Y. \& Setoyama, H. Structure of the intestinal flora responsible for development of the gut immune system in a rodent model. Microbes Infect. 2, 1343-1351 (2000).

73. Snel, J., Bakker, M.H. \& Heidt, P.J. Quantification of antigen-specific immunoglobulin A after oral booster immunization with ovalbumin in mice mono-associated with segmented filamentous bacteria or Clostridium innocuum. Immunol. Lett. 58, 25-28 (1997).

74. Macpherson, A.J. et al. A primitive T cell-independent mechanism of intestinal mucosal IgA responses to commensal bacteria. Science $\mathbf{2 8 8}$, 2222-2226 (2000).

75. Yoshida, M. et al. Human neonatal Fc receptor mediates transport of lgG into luminal secretions for delivery of antigens to mucosal dendritic cells. Immunity 20, 769-783 (2004).

76. Niess, J.H. et al. CX3CR1-mediated dendritic cell access to the intestinal lumen and bacterial clearance. Science 307, 254-258 (2005).

77. Cerutti, A. The regulation of IgA class switching. Nat. Rev. Immunol. 8, 421-434 (2008).

78. Tezuka, H. et al. Regulation of IgA production by naturally occurring TNF/ iNOS-producing dendritic cells. Nature 448, 929-933 (2007).

79. Uematsu, S. et al. Regulation of humoral and cellular gut immunity by lamina propria dendritic cells expressing Toll-like receptor 5. Nat. Immunol. 9, 769-776 (2008).

80. Tsuji, M. et al. Requirement for lymphoid tissue-inducer cells in isolated follicle formation and T cell-independent immunoglobulin A generation in the gut. Immunity 29, 261-271 (2008).

81. Bouskra, D. et al. Lymphoid tissue genesis induced by commensals through NOD1 regulates intestinal homeostasis. Nature 456, 507-510 (2008).

82. Happel, K.I. et al. Divergent roles of IL-23 and IL-12 in host defense against Klebsiella pneumoniae. J. Exp. Med. 202, 761-769 (2005).

83. Malley, R. et al. Antibody-independent, interleukin-17A-mediated, crossserotype immunity to pneumococci in mice immunized intranasally with the cell wall polysaccharide. Infect. Immun. 74, 2187-2195 (2006).

84. Mangan, P.R. et al. Transforming growth factor-beta induces development of the $T(H) 17$ lineage. Nature 441, 231-234 (2006).

85. Zenewicz, L.A. et al. Interleukin-22 but not interleukin-17 provides protection to hepatocytes during acute liver inflammation. Immunity $\mathbf{2 7}$, 647-659 (2007).

86. Ivanov, I.I. et al. The orphan nuclear receptor RORgammat directs the differentiation program of proinflammatory IL-17+ Thelper cells. Cell 126, 1121-1133 (2006)

87. McGeachy, M.J. \& Cua, D.J. Th17 cell differentiation: the long and winding road. Immunity 28, 445-453 (2008).

88. Veldhoen, M. et al. The aryl hydrocarbon receptor links TH17-cellmediated autoimmunity to environmental toxins. Nature 453, 106-109 (2008).

89. Zhou, L. et al. IL-6 programs $\mathrm{T}(\mathrm{H})-17$ cell differentiation by promoting sequential engagement of the IL-21 and IL-23 pathways. Nat. Immunol. 8, 967-974 (2007).

90. McGeachy, M.J. et al. TGF-beta and IL-6 drive the production of IL-17 and IL-10 by T cells and restrain $\mathrm{T}(\mathrm{H})-17$ cell-mediated pathology. Nat. Immunol. 8, 1390-1397 (2007).

91. Hue, S. et al. Interleukin-23 drives innate and T cell-mediated intestinal inflammation. J. Exp. Med. 203, 2473-2483 (2006).

92. Izcue, A. et al. Interleukin-23 restrains regulatory $T$ cell activity to drive $T$ cell-dependent colitis. Immunity 28, 559-570 (2008).

93. Duerr, R.H. et al. A genome-wide association study identifies IL23R as an inflammatory bowel disease gene. Science 314, 1461-1463 (2006).

94. Atarashi, K. et al. ATP drives lamina propria $\mathrm{T}(\mathrm{H}) 17$ cell differentiation. Nature 455, 808-812 (2008).

95. Ivanov, I.I. et al. Specific microbiota direct the differentiation of IL-17producing T-helper cells in the mucosa of the small intestine. Cell Host Microbe 4, 337-349 (2008).

96. Zaph, C. et al. Commensal-dependent expression of IL-25 regulates the IL-23-IL-17 axis in the intestine. J. Exp. Med. 205, 2191-2198 (2008).

97. Wen, L. et al. Innate immunity and intestinal microbiota in the development of type 1 diabetes. Nature 455, 1109-1113 (2008).

98. Hall, J.A. et al. Commensal DNA limits regulatory T cell conversion and is a natural adjuvant of intestinal immune responses. Immunity $\mathbf{2 9}$, 637-649 (2008). 


\section{REVIEW}

99. Ivanova, E.P., Alexeeva, Y.V., Pham, D.K., Wright, J.P. \& Nicolau, D.V. ATP level variations in heterotrophic bacteria during attachment on hydrophilic and hydrophobic surfaces. Int. Microbiol. 9, 37-46 (2006).

100. North, R.A. Molecular physiology of P2X receptors. Physiol. Rev. 82, 1013-1067 (2002).

101. Khakh, B.S. \& North, R.A. P2X receptors as cell-surface ATP sensors in health and disease. Nature 442, 527-532 (2006).

102. Mariathasan, S. et al. Cryopyrin activates the inflammasome in response to toxins and ATP. Nature 440, 228-232 (2006).

103. Li, M.O. \& Flavell, R.A. TGF-beta: a master of all T cell trades. Cell 134, 392-404 (2008).

104. Denning, T.L., Wang, Y.C., Patel, S.R., Williams, I.R. \& Pulendran, B. Lamina propria macrophages and dendritic cells differentially induce regulatory and interleukin 17-producing T cell responses. Nat. Immunol. 8, 1086-1094 (2007).

105. Sakaguchi, S., Yamaguchi, T., Nomura, T. \& Ono, M. Regulatory T cells and immune tolerance. Cell 133, 775-787 (2008).

106. Coombes, J.L. et al. A functionally specialized population of mucosal CD103+ DCs induces Foxp3+ regulatory T cells via a TGF-beta and retinoic acid-dependent mechanism. J. Exp. Med. 204, 1757-1764 (2007).
107. Sun, C.M. et al. Small intestine lamina propria dendritic cells promote de novo generation of Foxp3T reg cells via retinoic acid. J. Exp. Med. 204, 1775-1785 (2007).

108. Maynard, C.L. et al. Regulatory T cells expressing interleukin 10 develop from Foxp3+ and Foxp3 - precursor cells in the absence of interleukin 10. Nat. Immunol. 8, 931-941 (2007).

109. Rubtsov, Y.P. et al. Regulatory T cell-derived interleukin-10 limits inflammation at environmental interfaces. Immunity 28, 546-558 (2008).

110. Mazmanian, S.K., Round, J.L. \& Kasper, D.L. A microbial symbiosis factor prevents intestinal inflammatory disease. Nature 453, 620-625 (2008).

111. Artis, D. Epithelial-cell recognition of commensal bacteria and maintenance of immune homeostasis in the gut. Nat. Rev. Immunol. 8, 411-420 (2008).

112. Brandl, K. et al. Vancomycin-resistant enterococci exploit antibioticinduced innate immune deficits. Nature 455, 804-807 (2008).

113. Iwasaki, A. Mucosal dendritic cells. Annu. Rev. Immunol. 25, 381-418 (2007).

114. Pulendran, B., Tang, H. \& Denning, T.L. Division of labor, plasticity, and crosstalk between dendritic cell subsets. Curr. Opin. Immunol. 20, 61-67 (2008). 